\title{
Rikkunshito Increases Incretin Levels in Patients Underwent Pylorus-Preserving Pancreatico- Duodenectomy: A Case Control Study
}

Hiroshi Kono ( $\sim$ hkouno@yamanashi.ac.jp )

University of Yamanashi

Naohiro Hosomura

University of Yamanashi

Hidetake Amemiya

University of Yamanashi

Katsutoshi Shoda

University of Yamanashi

Shinji Furuya

University of Yamanashi

Hidenori Akaike

University of Yamanashi

Yoshihiko Kawaguchi

University of Yamanashi

Hiromichi Kawaida

University of Yamanashi

Daisuke Ichikawa

University of Yamanashi

\section{Research Article}

Keywords: gastrointestinal hormone, Japanese traditional herbal medicine, gastric inhibitory polypeptide, islet cells, insulin, incretin

Posted Date: January 27th, 2022

DOI: https://doi.org/10.21203/rs.3.rs-1176501/v1

License: (c) (1) This work is licensed under a Creative Commons Attribution 4.0 International License.

Read Full License 


\section{Abstract \\ Background}

Rikkunshito (TJ-43) improves the gastrointestinal disorders by increasing levels of active ghrelin which is belonging to gastrointestinal hormones; however, effects of TJ-43 on other gastrointestinal hormones are still unknown. Accordingly, the purpose of this study was to investigate effects of TJ-43 on other gastrointestinal hormones and incretins including gastric inhibitory polypeptide (GIP) and glucagon-like peptide-1 (GLP-1), which activate the islet cells in the pancreas.

\section{Methods}

Forty-one patients who underwent pylorus-preserving pancreatico-duodenectomy (PpPD) due to pancreato-biliary tumors were divided into two groups; patients were received TJ-43 (7.5 g daily dose) just after surgery [TJ-43(+) group, $n=21]$ or 3 weeks after surgery [TJ-43(-) group, $n=20$ ]. Blood samples were collected for analysis of regular clinical blood tests at designated time points. In addition, active and inactive form of ghrelin, spexin, cholecystokinin (CCK), peptide YY (PYY), gastric inhibitory peptide (GIP), and glucagon-like peptide-1 (GLP-1) levels were assayed by ELISA. One week after surgery, meals were starting and oral calorie intake was assessed both in the two groups after starting meals. Insulin secretion was also assessed.

\section{Results}

Active form of ghrelin levels significantly increased in the TJ-43(+) group compared with the TJ-43(-) group. Reflecting this result, oral intake of staple food (carbohydrate) significantly increased in the in the TJ-43(+) group. Levels of spexin slightly decreased after PpPD in the TJ-43(-) group and were not changed in the TJ-43(+) group before and after surgery. Levels of CCK and PYY were not different before and after surgery in the TJ-43(-) group; however, these levels increased significantly in the TJ-43(+) group. Alternatively, GIP and GLP-1 levels decreased in the TJ-43(-) group after PpPD. Importantly, these levels significantly increased in the TJ-43(+) group compared with the TJ-43(-) group. Although there were no significant differences between the TJ-43(+) group and the TJ-43 (-) group, insulin secretion increased in the $\mathrm{TJ}-43(+)$ group.

\section{Conclusions}

TJ-43 treatment increased expression of incretins in addition to active ghrelin. Thus, it could have advantage for oral food intake and blood sugar control in patients after pancreatic surgery.

\section{Background}


The Japanese herbal medicine "Rikkunshito" (TJ-43) is the form of extract of 8 crude herbal drugs (i.e., Atractylodis lanceae rhizoma, Ginseng radix, Pinelliae tuber, Hoelen, Zizyphi fructus, Aurantii nobilis pericarpium, Glycyrrhizae radix, and Zingiberis rhizoma). Hesperetin, hesperidin, naringenin, $3,3=, 4=, 5,6,7,8$-heptamethoxyflavone, nobiletin, tangeretin (Aurantii nobilis pericarpium), isoliquiritigenin, liquiritigenin, glycycoumarin (Glycyrrhizae radix), 8-shogaol, 10-shogaol, 10-dehydrogingerdin, 10 gingerdion, and 8-gingerol (Zingiberis rhizoma), which are typical components of TJ-43.

It was well known that TJ-43 increases expression of active form of ghrelin, which is an endogenous ligand of the growth hormone secretagogue receptor secreted mainly from the stomach [1]. Active form of ghrelin has an intense appetite-enhancing effect in addition to a growth hormone secretion-promoting effect [1], and is the only hormone which exhibits an appetite-promoting effect following peripheral intravenous treatment [2]. In addition, ghrelin has various actions including stimulation of secretion of growth hormone, gastric acid secretion [3], and induction of a positive energy balance [4]. The peripheral level of ghrelin is related to gastrointestinal disorders. Therefore, treatment of ghrelin has been a novel therapy for patients with anorexia-related gastrointestinal disorders [2]; however, the intravenous repeated administration of ghrelin clinically presents a considerable burden for patients.

Rikkunshito is used to treat upper gastrointestinal disorders [5]. Indeed, rats treated with TJ-43 enhanced gastric emptying [6]. Furthermore, treatment of TJ-43 in combination with an anti-emetic drug to patients with breast cancer prevented anorexia and emesis that occur as adverse reactions to chemotherapy [7]. Thus, TJ-43 prevents gastric motility and mobility. Takeda et al. previously reported that a flavonoid contained in TJ-43 suppressed a cisplatin-induced decrease in plasma active form of ghrelin levels and increased food intake in animal models [8]. Thus, one important effect of TJ-43 is increases in active form of ghrelin; however, effects of TJ-43 on other gastrointestinal hormones are not fully explored yet. Accordingly, the purpose of this study was to investigate effects of TJ-43 on gastrointestinal hormones, especially incretin including gastric inhibitory polypeptide (GIP) and glucagon-like peptide-1 (GLP-1), which activate the pancreatic islet cells and induce insulin secretion.

\section{Methods}

\section{Patients and sample collection}

Serum samples were obtained from 41 patients who underwent PpPD due to pancreato-biliary malignant tumors at the University of Yamanashi Hospital between 2015 and 2018 (Table 1). The histological diagnoses of the specimens were confirmed using the criteria of the uploaded World Health Organization classification. The stage was assessed according to the Union for International Cancer Control (UICC) classification, $7^{\text {th }}$ edition [9]. This study was approved by the Ethics Committee of University of Yamanashi (Chief of committee, Prof. Zentaro Yamagata; and Approval number: \#2211) and was performed in accordance with the ethical standards laid down in Declaration of Helsinki and its later amendments. Informed consent was obtained from all patients and/or donors of clinical samples including blood or tissues, where necessary, at the time of admission. 
Japanese traditional herbal medicine TJ-43 was treated with patient underwent gastrointestinal surgery including pancreatic surgery [10]. Based on treatments of TJ-43, patients were divided into two groups after surgery, $₫:$ TJ-43(-) group ( $n=20, T J-43$ (7.5g/day) was treated from post-operative day (POD) 21 via

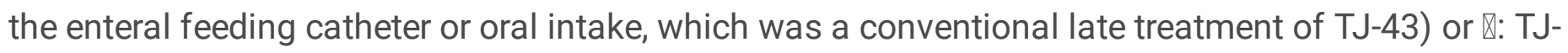
$43(+)$ group $(n=21, T J-43(7.5 \mathrm{~g} /$ day $)$ was treated daily from POD 1 via the enteral feeding catheter or oral intake, which was an improved early treatment of TJ-43) (Figure 1). No significant differences in clinical features were observed between the TJ-43(-) group and TJ-43(+). Rikkunshito (supplied by Tsumura \& Co., Tokyo, Japan) is a mixture of eight herbal plants, as shown in Table 2. Enteral nutrition ( $900 \mathrm{Kcal} /$ day) was started at POD 1 via the enteral feeding catheter and was continued during the observation period in this study. In addition, 7 days after surgery, the post-operative meal was started. Furthermore, total oral calorie intake from diets [staple food (mainly carbohydrates) and side dish (protein/fat)] were assessed both in the two groups (at POD 21).

\section{Blood samples}

Blood samples were collected before surgery and 1,2 and 3 weeks after surgery for regular clinical blood tests in the hospital. In addition, serum and plasma samples were also stored at $-80^{\circ} \mathrm{C}$ until further measurements.

\section{Insulin secretion}

Immunoreactive insulin (IRI) was measured by clinical laboratory analysis in the our hospital.

\section{Measurement of ghrelin and other gastrointestinal hormones}

Plasma acyl (active form) and desacyl (inactive form) ghrelin levels were measured by enzyme-linked immuno sorbent assay (ELISA) kits (Iwai Chemicals Co., Tokyo, Japan).

Serum spexin (LifeSpan BioSciences, Inc., Seattle, WA), cholecystokinin (CCK) (LifeSpan BioSciences), peptide YY (PYY) (LifeSpan BioSciences), gastric inhibitory peptide (GIP; or glucose-dependent insulinotropic polypeptide) (RayBiotech Life Inc., Peachtree Corners, GA), and glucagon-like peptide-1 (GLP-1) (Invitrogen, Waltham, MA) levels were also measured by ELISA.

\section{Statistical analysis.}

All statistical analyses were conducted with EZR (Saitama Medical Center, Jichi Medical University, Saitama, Japan), which is a graphical user interface for R (The R Foundation for Statistical Computing, Vienna, Austria) [11].

Data are expressed as the mean \pm standard error of the mean (SEM). Comparisons between the groups were assessed using the paired $t$-test or ANOVA with Bonferroni's post-hoc test. $P<0.05$ was considered significant. 


\section{Results}

\section{Treatment with TJ-43 after pancreatic surgery.}

No significant differences were observed in clinical features between the two groups (Table 1). Furthermore, the patients treated with TJ-43 did not show any complications after treatment.

\section{Effect of TJ-43 on plasma ghrelin levels.}

Desacyl (inactive form) and acyl (activate form) ghrelin was detected in plasma collected from patients before surgery [acyl ghrelin: TJ-43(-) group; $13.6 \pm 1.5 \mathrm{fmol} / \mathrm{ml}$ and TJ-43(+) group; $12.2 \pm 1.9 \mathrm{fmol} / \mathrm{ml}$, and desacyl ghrelin: TJ-43(-) group; $104.2 \pm 7.5 \mathrm{fmol} / \mathrm{ml}$ and TJ-43(+) group; $103.4 \pm 7.6 \mathrm{fmol} / \mathrm{ml}$ (mean \pm SEM)]. The plasma desacyl ghrelin levels were slightly decreased in both two groups after surgery [TJ43(-) group; $97.8 \pm 6.9 \mathrm{fmol} / \mathrm{ml}$, and TJ-43(+) group; $98.4 \pm 7.1 \mathrm{fmol} / \mathrm{ml}$, consistent with a previous report [12]. Increases in active form ghrelin were minimal in the TJ-43(-) group $(14.3 \pm 0.9 \mathrm{fmol} / \mathrm{ml})$; however, they were significantly greater in the TJ-43(+) group compared with values before surgery $(23.7$ $\pm 1.9 \mathrm{fmol} / \mathrm{ml}$ ) [1] [8] [12].

\section{Effect of TJ-43 on consumption of meals.}

Reflecting the results of active form ghrelin levels, total oral dietary calorie intake was greater in the TJ43(+) group (491.5 $\pm 59.2 \mathrm{Kcal})$ compared with the TJ-43(-) group (317.5 $\pm 52.3 \mathrm{Kcal})$ (Fig. 2).

Furthermore, calorie intake from staple food (mainly carbohydrate) was significantly greater in the TJ$43(+)$ group $(375.3 \pm 62.3 \mathrm{Kcal})$ compared with the TJ-43(-) group $(236.9 \pm 49.7 \mathrm{Kcal})$, consistent with a previous report [12].

\section{Effect of TJ-43 on serum gastrointestinal hormone levels.}

Gastrointestinal hormones including spexin, CCK and PYY were detected in serum collected prior to surgery (Fig. 3). The levels of spexin slightly decreased in patients underwent PpPD both in the two groups. Furthermore, levels of CCK and PYY were not significantly different before and after PpPD in the TJ-43(-) group; however, in the TJ-43(+) group, these levels significantly increased compared with the TJ43(-) group after surgery.

\section{Effect of TJ-43 on serum incretin hormones.}

In the TJ-43(-) group, serum GIP and GLP-1 levels decreased after PpPD compared with before surgery. In contrast, values were significantly greater in the TJ-43(-) group compared with those in the TJ-43(-) group after surgery (Fig. 4). Furthermore, these increases were more significant in values of GIP compared with those of GLP-1.

\section{Effect of TJ-43 on IRI levels.}


Immunoreactive insulin levels were measured at 3 weeks after surgery. Although there were no significant differences in IRI levels between the two groups, it was greater in the TJ-43(+) group compared with the TJ-43(-) group (Fig. 5).

\section{Discussion}

\section{Effects of TJ-43 on expression of ghrelin and food intake after pancreatico-duodenectomy.}

Ghrelin and its receptor are produced and expressed in the hypothalamus arcuate nucleus neurons and the stomach. Peripheral or intracerebral administration of ghrelin to animals enhances gastrointestinal motility and mobility and increases food intake [13]. Furthermore, increases in active ghrelin level were reported in functional dyspepsia [13], chronic gastritis and gastric ulcer [14]. Thus, ghrelin is associated with gastrointestinal physiology and diseases. Rikkunshito increases release of active ghrelin from the intestine [1]. Furthermore, TJ-43 improved cisplatin-induced decreases in plasma acyl ghrelin level and food intake in rats. In the present study, early treatment of TJ-43 after surgery also increased plasma acyl ghrelin levels, confirming previous reports that TJ-43 increases systemic active ghrelin. This increase may improve food intake after gastrointestinal surgery.

It is well known that postoperative DGE is the common complication after PpPD. This complication can lead to epigastric discomfort, loss of appetite, prolonged hospitalization [15]. In the present study, acyl ghrelin levels increased in the TJ-43(+) group compared with the TJ-43(-) group after PpPD. Since the only difference between the two groups was when starting TJ-43, the reason of the increase in active ghrelin levels was most likely treatment of TJ-43, as reported previously [1] [12] [16]. Furthermore, carbohydrate intake significantly increased in the TJ-43(+) group compared with the TJ-43(-) group after PpPD (Fig. 2). Thus, TJ-43 most likely improves DGE by increase in active ghrelin levels after PpPD, consistent with the previous reports [1] [8] [12].

\section{Effects of TJ-43 on expression of gastrointestinal hormones.}

The gastrointestinal hormones are polypeptides which are produced from endocrine cells in the stomach, pancreas and small intestine, and controlling functions including gastrointestinal motility, secretion, absorption, growth, and development of the digestive organs [17]. The gastrointestinal hormones are also found in the enteric nervous system and the central nervous system. It was reported from the previous studies that most of the gut-derived peptides also act as neurotransmitters and neuromodulators in the central and peripheral nervous systems [17].

In this study, serum levels of gastrointestinal hormone including spexin, CCK and PYY were detected in patients following PpPD (Fig. 3). Spexin expression was not markedly changed both in the two groups. Furthermore, expressions of CCK and PYY were increased in the TJ-43(+) group after surgery. Indeed, the blood concentration of PYY increases after food intake and decreases by fasting [18], and has been shown to reduce appetite. It inhibits gastric motility and increases water and electrolyte absorption in the large intestine [19], and also suppress pancreatic secretion. In animal studies, vasoactive intestinal 
polypeptide stimulated PYY release [20], while gastrin inhibited basal and meal-stimulated PYY levels [21]. Thus, PYY has an opposing clinical effect to ghrelin. Comparing the effects of TJ-43 on levels of active ghrelin and PYY, the increment of ghrelin was greater than that of PYY, suggesting that effect of ghrelin induced by TJ-43 treatment may have more strong compared with those of PYY.

Cholecystokinin which stimulates PYY release, is produced in the endocrine I cell of the mucosa of the duodenum and the jejunum [22]. In humans, the physiologic functions of CCK include the ability to stimulate gallbladder contraction, increase pancreatic enzyme and insulin secretion, and regulate food intake and gastrointestinal motility [23]. In this study, CCK was also affected by TJ-43 treatment after pancreatic surgery (Fig. 3). Since CCK increases pancreatic enzyme and insulin secretion insulin secretion, TJ-43 may be valuable after pancreatic resection.

\section{Effects of TJ-43 on incretin expression.}

Incretins are gastrointestinal polypeptide hormones secreted after nutrient intake. They stimulate insulin secretion from the islet $\beta$ cell in a blood glucose-dependent manner, suggesting that hypoglycemia is hardly happened without meal consumption. In addition, it inhibits gastric acid secretion, but has no effect on gastric emptying [24]. One of incretin GIP is secreted from the $K$ cells existed in the upper intestine, including the duodenum and jejunum. Another incretin GLP-1 is secreted from the L cells in the lower intestine and also increases activation of the islet cell and secretion of insulin. In the present study, plasma GIP and GLP-1 levels were increased by TJ-43 treatment in patients underwent PpPD (Fig. 4). Furthermore, insulin secretion increased by TJ-43 treatment (Fig. 2), although there were no significant differences. In addition, in the pilot study using an animal model, serum and intestinal expression of GIP and GLP-1 also significantly increased, and insulin expression in the pancreatic islet was markedly enhanced after continuous TJ-43 administration by gavage (unpublished data). Thus, TJ-43 can increase incretin and insulin expression.

\section{Conclusion}

Taken together, TJ-43 may have an advantage for oral dietary intake and blood sugar control most likely by increasing expression of incretin in addition to active ghrelin after pancreatic surgery, which reduces the total volume of pancreas tissues.

\section{Abbreviations}

TJ-43, Rikkunshito; PpPD, pylorus-preserving pancreaticoduodenectomy; DGE, delayed gastric emptying; PYY, peptide YY; GIP, gastric inhibitory peptide; GLP-1, glucagon-like peptide-1; CCK, cholecystokinin; IRI, immunoreactive insulin; ELISA, enzyme-linked immuno sorbent assay; UICC, Union for International Cancer Control; Ph, pancreas head, IPMC, intraductal papillary mucinous carcinoma; IPMA, intraductal papillary mucinous adenoma; CBD, common bile duct; $\mathrm{Ca}$, carcinoma; NET, neuroendocrine tumor; $\mathrm{GB}$, gall bladder; $\mathrm{Hb}$, hemoglobin; and JP, The Japanese Pharmacopoeia. 


\section{Declarations}

\section{Ethics approval and consent to participate}

This study was approved by the Ethics Committee of University of Yamanashi (Chief of committee, Prof. Zentaro Yamagata; and Approval number: \#2211) and was performed in accordance with the ethical standards laid down in Declaration of Helsinki and its later amendments. Informed consent was obtained from all patients and/or donors of clinical samples including blood or tissues, where necessary, at the time of admission.

\section{Consent for publication}

Not applicable

\section{Availability of data and materials}

The datasets used and/or analyzed during the current study are available from the corresponding author on reasonable request.

\section{Competing interests}

The authors declare that they have no competing interests.

\section{Funding}

The authors declare that there are no funds on this study.

\section{Authors' contributions}

Kono, H., conducting and organizing this experiment; Hosomura, N., assessment of samples; Amemiya, H., assessment of samples; Shoda K., analyzing the data; Furuya, S., analyzing the data; Akaike, H., assessment of samples; Kawaguchi, Y, analyzing the data; Kawaida, H., collecting samples; and Ichikawa, D., advising for this experiment

\section{Acknowledgements}

Not applicable

\section{References}

1. Kojima M, Hosoda H, Date Y, Nakazato M, Matsuo H, Kangawa K: Ghrelin is a growth-hormonereleasing acylated peptide from stomach. Nature 1999, 402(6762):656-660.

2. Perchard R, Clayton PE: Ghrelin and Growth. Endocr Dev 2017, 32:74-86. 
3. Ibrahim Abdalla MM: Ghrelin - Physiological Functions and Regulation. Eur Endocrinol 2015, 11(2):90-95.

4. Muller TD, Nogueiras R, Andermann ML, Andrews ZB, Anker SD, Argente J, Batterham RL, Benoit SC, Bowers CY, Broglio F et al: Ghrelin. Mol Metab 2015, 4(6):437-460.

5. Kawahara H, Kubota A, Hasegawa T, Okuyama H, Ueno T, Ida S, Fukuzawa M: Effects of rikkunshito on the clinical symptoms and esophageal acid exposure in children with symptomatic gastroesophageal reflux. Pediatr Surg Int 2007, 23(10):1001-1005.

6. Kido T, Nakai Y, Kase Y, Sakakibara I, Nomura M, Takeda S, Aburada M: Effects of rikkunshi-to, a traditional Japanese medicine, on the delay of gastric emptying induced by N(G)-nitro-L-arginine. J Pharmacol Sci 2005, 98(2):161-167.

7. Tomono H, Ito $\mathrm{Y}$, Watanabe T: [Successful antiemetic treatment of TSUMURA Rikkunshi-to Extract Granules for ethical use in addition to other antiemetic agents in neoadjuvant chemotherapy for an advanced breast cancer patient]. Gan To Kagaku Ryoho 2006, 33(8):1129-1131.

8. Hattori T, Yakabi K, Takeda H: Cisplatin-induced anorexia and ghrelin. Vitam Horm 2013, 92:301317.

9. Luttges J: [What's new? The 2010 WHO classification for tumours of the pancreas]. Pathologe 2011, 32 Suppl 2:332-336.

10. Takiguchi S, Hiura Y, Takahashi T, Kurokawa Y, Yamasaki M, Nakajima K, Miyata H, Mori M, Hosoda $\mathrm{H}$, Kangawa $\mathrm{K}$ et al: Effect of rikkunshito, a Japanese herbal medicine, on gastrointestinal symptoms and ghrelin levels in gastric cancer patients after gastrectomy. Gastric Cancer 2013, 16(2):167-174.

11. Kanda Y: Investigation of the freely available easy-to-use software 'EZR' for medical statistics. Bone Marrow Transplant 2013, 48(3):452-458.

12. Takeda H, Muto S, Nakagawa K, Ohnishi S, Asaka M: Rikkunshito and ghrelin secretion. Curr Pharm Des 2012, 18(31):4827-4838.

13. Shinomiya T, Fukunaga M, Akamizu T, Irako T, Yokode M, Kangawa K, Nakai Y, Nakai Y: Plasma acylated ghrelin levels correlate with subjective symptoms of functional dyspepsia in female patients. Scand J Gastroenterol 2005, 40(6):648-653.

14. Isomoto H, Ueno H, Nishi Y, Yasutake T, Tanaka K, Kawano N, Ohnita K, Mizuta Y, Inoue K, Nakazato $\mathrm{M}$ et al: Circulating ghrelin levels in patients with various upper gastrointestinal diseases. Dig Dis Sci 2005, 50(5):833-838.

15. Balzano G, Zerbi A, Braga M, Rocchetti S, Beneduce AA, Di C, V: Fast-track recovery programme after pancreatico- duodenectomy reduces delayed gastric emptying. Br J Surg 2008, 95(11):1387-1393.

16. Takeda H, Sadakane C, Hattori T, Katsurada T, Ohkawara T, Nagai K, Asaka M: Rikkunshito, an herbal medicine, suppresses cisplatin-induced anorexia in rats via 5-HT2 receptor antagonism. Gastroenterology 2008, 134(7):2004-2013.

17. Johnson LR: Gastrointestinal hormones and their functions. Annu Rev Physiol 1977, 39:135-158. 
18. Murphy KG, Bloom SR: Gut hormones and the regulation of energy homeostasis. Nature 2006, 444(7121):854-859.

19. Liu CD, Aloia T, Adrian TE, Newton TR, Bilchik AJ, Zinner MJ, Ashley SW, McFadden DW: Peptide YY: a potential proabsorptive hormone for the treatment of malabsorptive disorders. Am Surg 1996, 62(3):232-236.

20. Ballantyne GH: Peptide YY(1-36) and peptide YY(3-36): Part I. Distribution, release and actions. Obes Surg 2006, 16(5):651-658.

21. Sachs G, Zeng N, Prinz C: Physiology of isolated gastric endocrine cells. Annu Rev Physiol 1997, 59:243-256.

22. Polak JM, Bloom SR, Rayford PL, Pearse AG, Buchan AM, Thompson JC: Identification of cholecystokinin-secreting cells. Lancet 1975, 2(7943):1016-1018.

23. Mutt V: Further investigations of intestinal hormonal polypeptides. Clin Endocrinol (Oxf) 1976, 5 Suppl:175S-183S.

24. Nauck MA, Meier JJ: Incretin hormones: Their role in health and disease. Diabetes Obes Metab 2018, 20 Suppl 1:5-21.

\section{Tables}


Table 1

Clinicopathological characteristics

\begin{tabular}{|c|c|c|c|c|}
\hline Variable & & TJ43-(n = 20) & TJ43区(n = 21) & $P$ \\
\hline Age & years & $67 \pm 7.0$ & $66 \pm 7.7$ & 0.962 \\
\hline \multirow[t]{2}{*}{ Sex } & Male & $10(50 \%)$ & $14(67 \%)$ & 0.199 \\
\hline & Female & $10(50 \%)$ & $7(33 \%)$ & \\
\hline \multirow[t]{7}{*}{ Disease } & Pancreas Ca. (Ph) & $7(35 \%)$ & $8(38 \%)$ & \\
\hline & IPMC & $2(10 \%)$ & $2(10 \%)$ & \\
\hline & IPMA & $2(10 \%)$ & $1(5 \%)$ & \\
\hline & CBD Ca. & $5(25 \%)$ & $5(24 \%)$ & \\
\hline & Vater Ca. & $3(15 \%)$ & $4(19 \%)$ & \\
\hline & Panaceas-NET & $1(5 \%)$ & $0(0 \%)$ & \\
\hline & GB Ca. & $0(0 \%)$ & $1(5 \%)$ & \\
\hline UICC Tumor stage & Pancreas Ca. (Ph) & $(0 / 0 / 1 / 6)$ & $(0 / 0 / 3 / 5)$ & \\
\hline Pancreas Ca. & IPMC & $(1 / 0 / 1 / 0)$ & $(0 / 0 / 1 / 1)$ & \\
\hline (0 / I/ IIA/ IIB) & IPMA & $\mathrm{N} / \mathrm{A}$ & $\mathrm{N} / \mathrm{A}$ & \\
\hline Other Ca. & CBD Ca. & $(0 / 1 / 4 / 0)$ & $(0 / 3 / 2 / 0)$ & \\
\hline \multirow[t]{3}{*}{$(0 / \mathrm{I} / \mathrm{II} / \mathrm{III})$} & Vater Ca. & $(0 / 1 / 2 / 0)$ & $(0 / 2 / 2 / 0)$ & \\
\hline & P-NET & $(0 / 1 / 0 / 0)$ & $\mathrm{N} / \mathrm{A}$ & \\
\hline & GB Ca. & $\mathrm{N} / \mathrm{A}$ & $(0 / 0 / 1 / 0)$ & \\
\hline \multirow{7}{*}{$\begin{array}{l}\text { Tumor differentiation } \\
\text { (well/mod/poor) }\end{array}$} & Pancreas Ca. (Ph) & $(3 / 2 / 2)$ & $(1 / 6 / 1)$ & \\
\hline & IPMC & $(0 / 2 / 0)$ & $(1 / 0 / 1)$ & \\
\hline & IPMA & $\mathrm{N} / \mathrm{A}$ & $\mathrm{N} / \mathrm{A}$ & \\
\hline & CBD Ca. & $(0 / 5 / 0)$ & $(2 / 2 / 1)$ & \\
\hline & Vater Ca. & $(2 / 1 / 0)$ & $(1 / 3 / 0)$ & \\
\hline & P-NET & $\mathrm{N} / \mathrm{A}$ & $\mathrm{N} / \mathrm{A}$ & \\
\hline & GB Ca. & N/A & $(0 / 1 / 0)$ & \\
\hline Time of operation & minutes & $500 \pm 56$ & $509 \pm .72$ & 0.299 \\
\hline Blood loss & $\mathrm{ml}$ & $692 \pm .54$ & $959 \pm .66$ & 0.182 \\
\hline $\mathrm{HbA} 1 \mathrm{c}$ & $\%$ & $5.6 \pm 2.2$ & $5.7 \pm 2.3$ & 0.892 \\
\hline
\end{tabular}




\begin{tabular}{|c|c|c|c|c|}
\hline Variable & & TJ43-(n = 20) & TJ43区(n = 21) & $P$ \\
\hline \multirow[t]{2}{*}{ Tumor markers } & CEA (ng/ml) & $3.1 \pm 1.3$ & $3.7 \pm 1.1$ & 0.872 \\
\hline & CA19-9 (U/ml) & $455 \pm 23$ & $451 \pm 29$ & \\
\hline
\end{tabular}

Table 2

Composition of Rikkunshito

\begin{tabular}{|l|c|}
\hline JP Atractylodes Lancea Rhizome & $\mathbf{4 . 0} \mathbf{~ g}$ \\
\hline JP Ginseng & $4.0 \mathrm{~g}$ \\
\hline JP Pinellia Tuber & $4.0 \mathrm{~g}$ \\
\hline JP Poria Sclerotium & $4.0 \mathrm{~g}$ \\
\hline JP Jujube & $2.0 \mathrm{~g}$ \\
\hline JP Citrus Unshiu Peel & $2.0 \mathrm{~g}$ \\
\hline JP Glycyrrhiza & $1.0 \mathrm{~g}$ \\
\hline JP Ginger & $0.5 \mathrm{~g}$ \\
\hline JP, The Japanese Pharmacopoeia. & \\
7.5 g of TSUMURA Rikkunshito extract granules contains $4.0 \mathrm{~g}$ of a dried extract of the following \\
mixed crude drugs.
\end{tabular}

\section{Figures}




\section{Operation \\ $\downarrow$}

$\begin{array}{llll}0 & 1 & 2 & 3 \text { weeks }\end{array}$

blood sampling

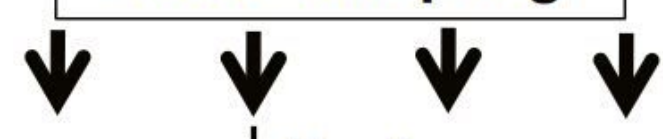

Meals

TJ-43 -

Rikkunshito treatment

TJ-43+

Rikkunshito treatment

Figure 1

Study protocol.

Study protocol are shown in Figure 1. TJ-43, Rikkunshito. 


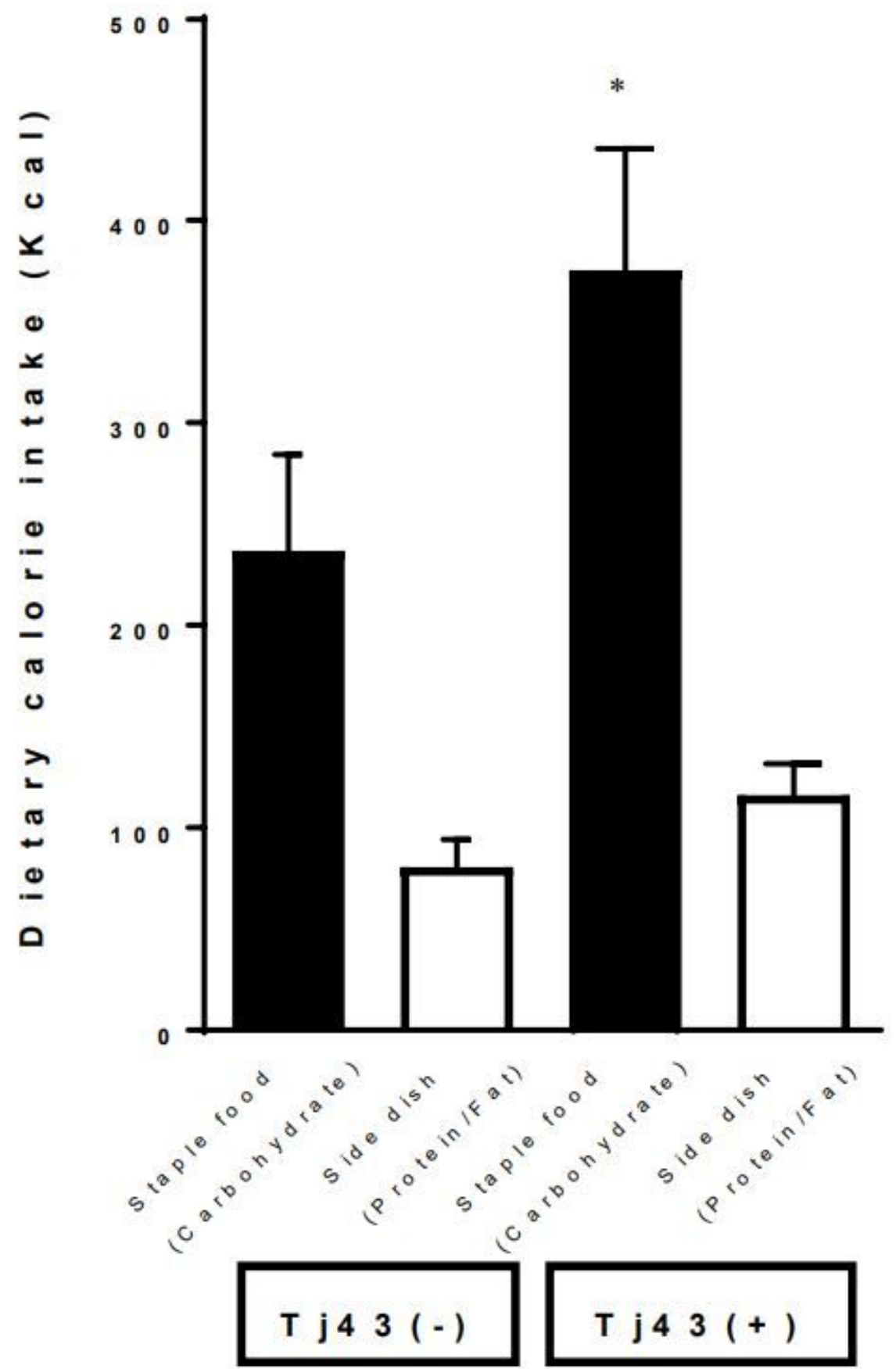

Figure 2

Oral dietary intake after pancreatic surgery.

After starting meals, the amount of oral dietary intake of each meal was scored from 0 to $100 \%$ (staple food and side dishes), and the average oral intake for seven days (POD 21) was calculated and analyzed 
in the two groups. TJ-43, Rikkunshito. *, $\mathrm{P}<0.05$ compared with carbohydrate intake in the TJ-43(-) group by ANOVA with Bonferroni's post-hoc test.

A

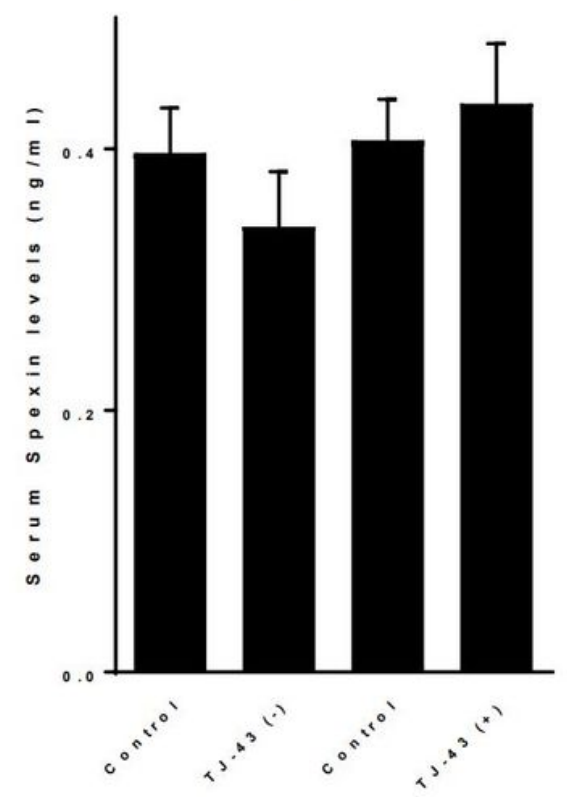

B

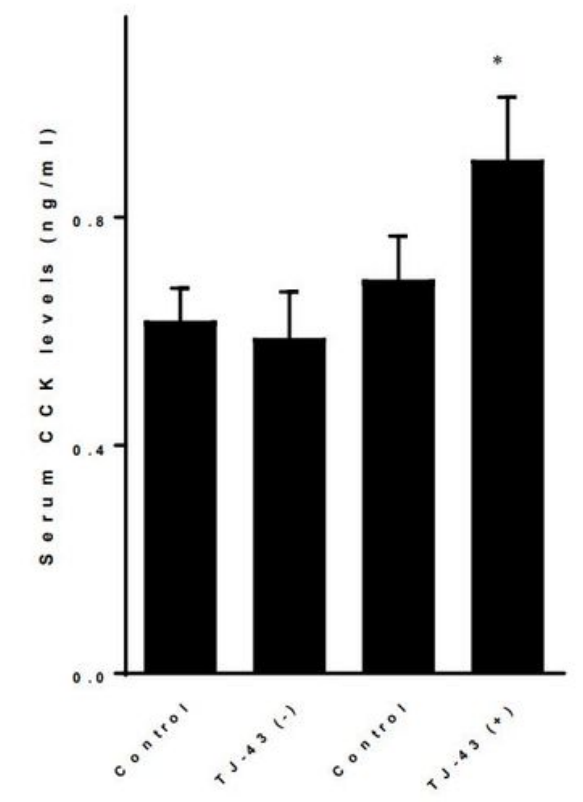

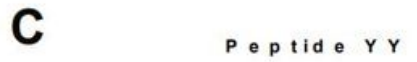

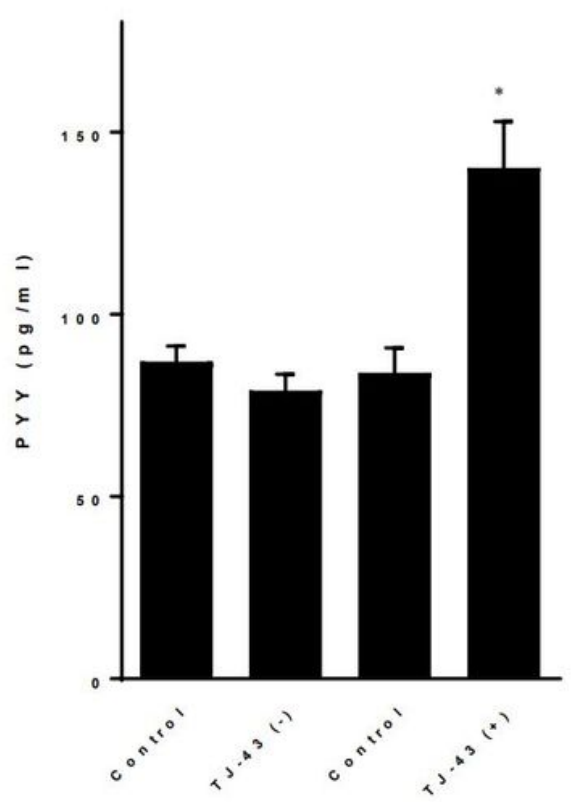

Figure 3

Serum spexin, CCK and PYY levels.

Serum levels of spexin (A), CCK (B), and PYY (C) were measured by ELISA. Control levels were values before surgery. *, $P<0.05$ compared with the TJ-43(-) group by ANOVA with Bonferroni's post-hoc test. TJ43, Rikkunshito; CCK, cholecystokinin; and PYY, peptide YY. 
A

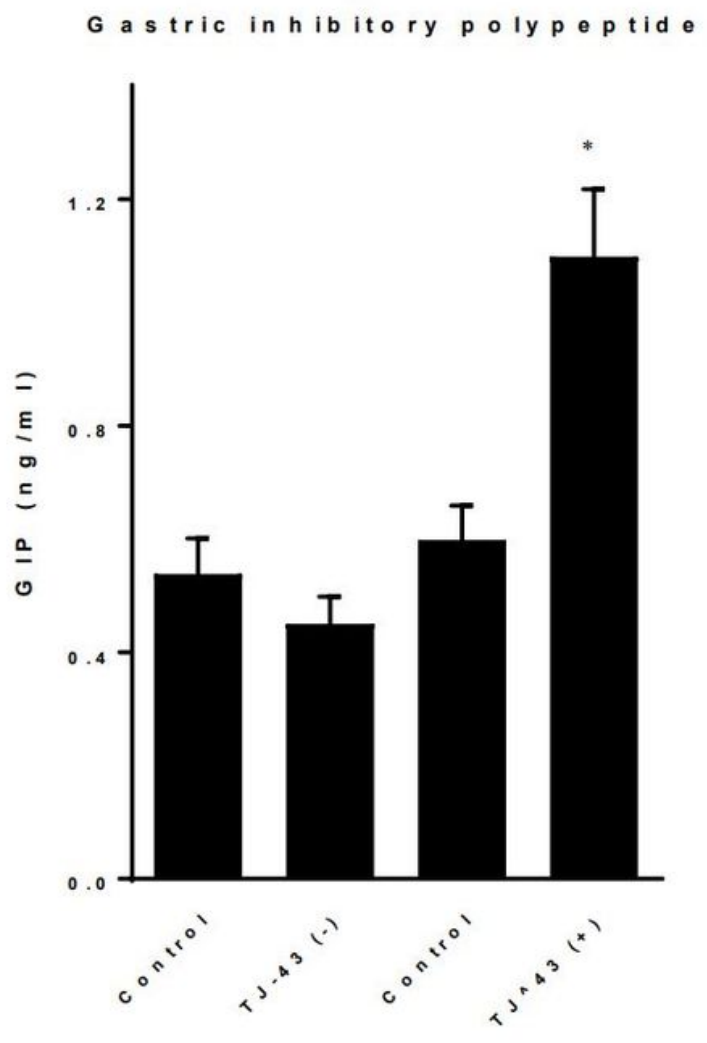

B

G Iucagon-like polypeptide-1

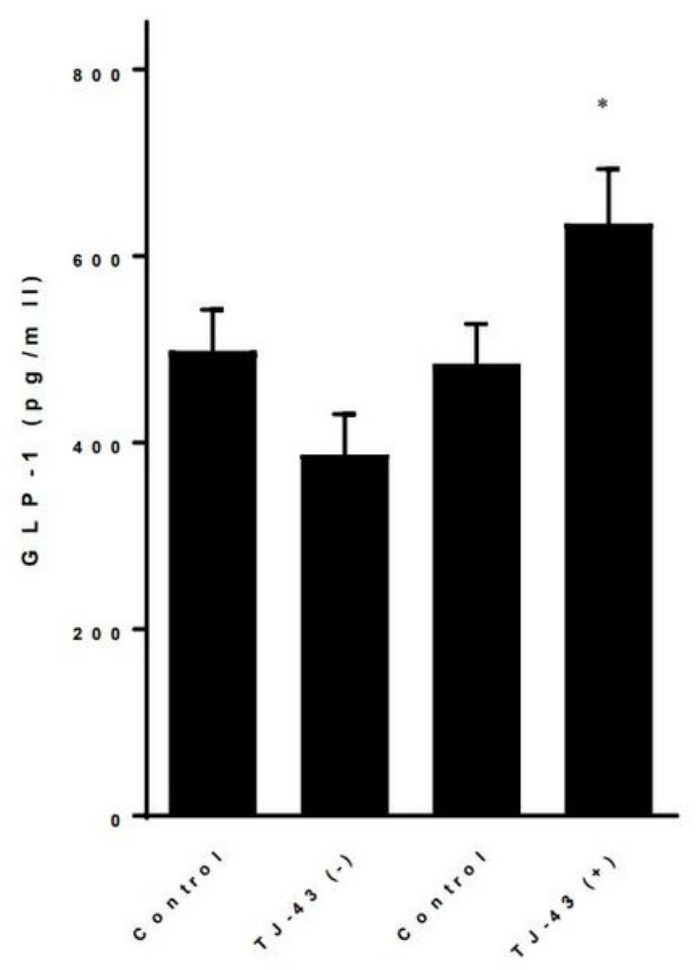

Figure 4

Serum incretin levels.

Serum levels of gastric inhibitory peptide (A) and glucagon-like polypeptide-1 (B) were measured by ELISA. Control levels were values before surgery. TJ-43, Rikkunshito; GIP, gastric inhibitory peptide; and GLP-1, glucagon-like polypeptide-1. *, $P<0.05$ compared with the TJ-43(-) group by ANOVA with Bonferroni's post-hoc test. 


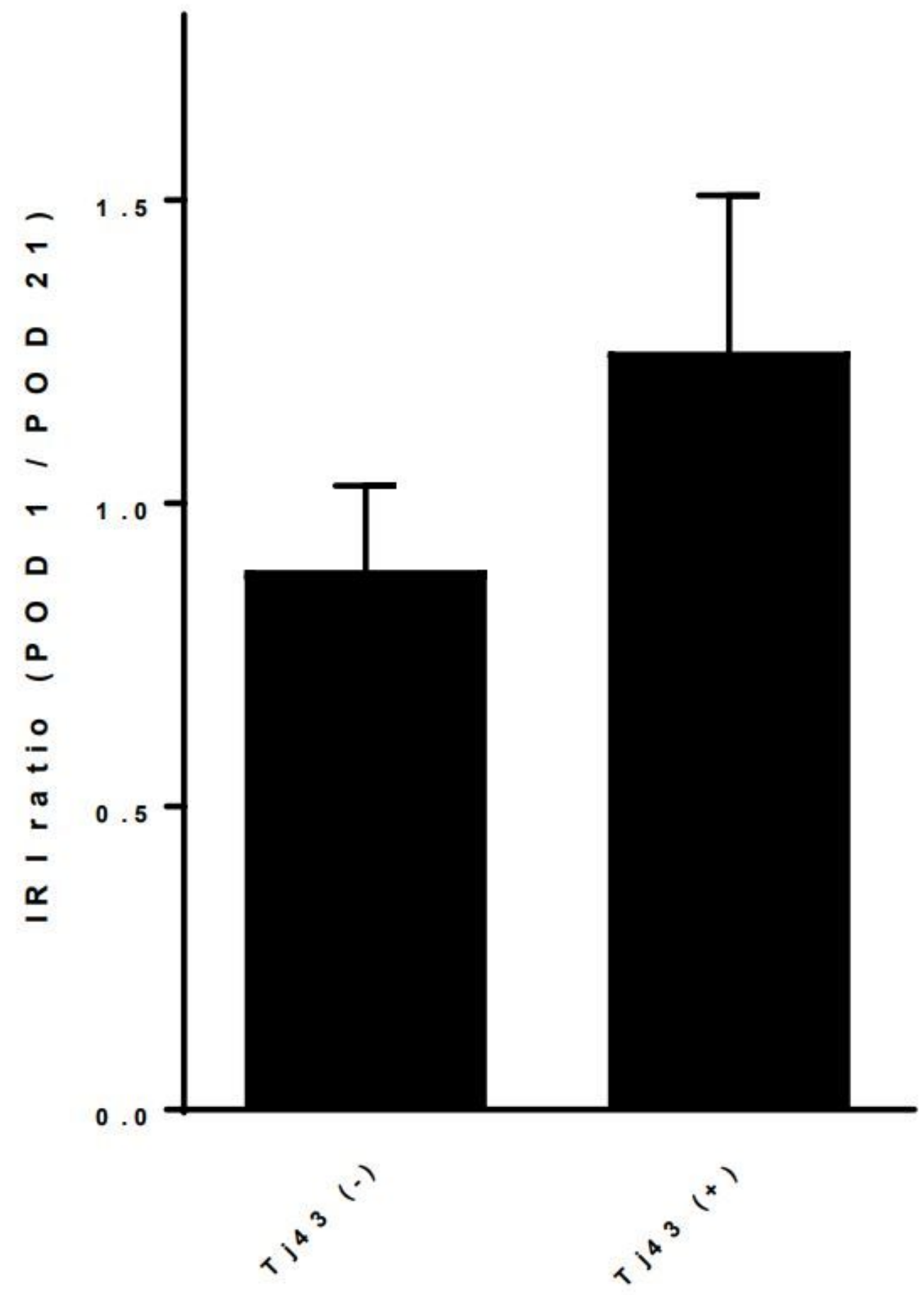

Figure 5

Effect of TJ-43 on insulin secretion.

Immunoreactive insulin levels are shown. TJ-43, Rikkunshito; and IRI, Immunoreactive insulin *, $P<0.05$ compared with the TJ-43(-) group by ANOVA with Bonferroni's post-hoc test. 